Cahiers d'études japonaises

16 | 2009

L'invention des « arts populaires » - Yanagi Sōetsu et le Mingei

\title{
Bibliographie du dossier L'invention des « arts populaires »
}

Jean-Michel Butel, Damien Kunik, Michael Lucken, François Macé, Christophe Marquet et Laurent Nespoulous

\section{(2) OpenEdition}

Journals

Édition électronique

URL : https://journals.openedition.org/cipango/369

DOI : $10.4000 /$ cipango.369

ISSN : 2260-7706

Éditeur

INALCO

Édition imprimée

Date de publication : 1 janvier 2009

Pagination : 157-169

ISSN : 1164-5857

Référence électronique

Jean-Michel Butel, Damien Kunik, Michael Lucken, François Macé, Christophe Marquet et Laurent Nespoulous, «Bibliographie du dossier L'invention des « arts populaires » », Cipango [En ligne], 16। 2009, document 12, mis en ligne le 22 novembre 2011, consulté le 30 juin 2021. URL : http:// journals.openedition.org/cipango/369; DOI : https://doi.org/10.4000/cipango.369

Ce document a été généré automatiquement le 30 juin 2021.

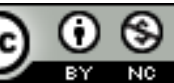

Cipango est mis à disposition selon les termes de la Licence Creative Commons Attribution - Pas d'Utilisation Commerciale 4.0 International. 


\title{
Bibliographie du dossier L'invention des « arts populaires »
}

\author{
Jean-Michel Butel, Damien Kunik, Michael Lucken, François Macé, \\ Christophe Marquet et Laurent Nespoulous
}

1 AKASHI Someto 明石染人, 1929 《Mingei to shite no ema no kōsatsu 》 民藝としての絵 馬の考察 (Les plaquettes votives vues sous l'angle de l'art populaire), Bi 美, vol. 23, $\mathrm{n}^{\circ}$ 4-5, Kyōto, Unsōdō.

2 AKIYAMA Kikuo 秋山喜久夫, 1973 Koi Enkū. Ōmiya zakki-chō 1 恋円空 一大宮䧱記帳1 (Enkū amoureux. Cahiers d'Ōmiya 1), Urawa, Saitama-ken Kyōdo shiryō kankōkai 埼玉 県郷土史料刊行会.

3 ARISHIMA Takeo 有島武郎, 1922 《Sengen hitotsu »宣言一つ, Kaizō 改造, janvier 1922 ; repris dans Arishima Takeo zenshū 有島武郎全集, vol. 9, Tōkyō, Chikuma shobō 筑摩書 房, 1981.

4 Arts \& Crafts from Morris to Mingei, Musée d'art de la ville de Tōkyō (Tōkyō-to bijutsukan 東京都美術館), 2009.

5 ASANO Tōru, 1986 « La peinture à l'époque Taishō ", in Japon des avant-gardes, Paris, Éditions du Centre Pompidou, p. 50-63.

6 ASO Noriko, 2009 "Mediating the Masses: Yanagi Sōetsu and Fascism », in Alan Tansman, Marilyn Ivy (éd.), The Culture of Japanese Fascism, Durham, Duke University Press, p. 139-154.

7 BENJAMIN Walter, (1935) 2000 L'CEuvre d'art à l'époque de sa reproductibilité technique, traduction de Rainer Rochlitz, in CEuvres III, Paris, Gallimard, p. 67-113.

8 BLANCHETIERE François, 2007 "Don et contre-don : Rodin et le groupe Shirakaba », in Rodin. Le rêve japonais, Paris, Musée Rodin / Flammarion, p. 195-219.

9 BOUchy Anne, 2003 "Une voie de l'“art premier” dans le Japon du XVII ${ }^{\mathrm{e}}$ siècle. La statuaire d'Enkū, le pérégrin de l'Essentiel », L'Homme, n 165, p. 143-172.

10 BRANDT Kim, 2007 Kingdom of Beauty. Mingei and the Politics of Folk Art in Imperial Japan, Durham, Duke University Press, 306 p. 
11

Butsuzō. Ichiboku ni komerareta inori 仏像 一一木にこめられた祈り (Shaping Faith Japanese Ichiboku Buddhist Statues), Tōkyō kokuritsu hakubutsukan 東京国立博物館, Yomiuri shinbun, 2006.

cHо Cha-yong, 1982 Guardians of Happiness. Shamanistic Tradition in Korean Folk Painting, Séoul, Emileh Museum, $91 \mathrm{p}$.

CHO Cha-yong 趙子庸, KIM Ch'ŏl-sun 金哲淳, 1989 Chosŏn sidae minhwa 朝鮮時代民畫

(La peinture populaire de l'époque Chosŏn), Séoul, Yegyŏng Sanŏpsa 예 경산업사, 304 p.

CHO Cha-yong, LEE U-fan, 1990 Traditional Korean Painting. A Lost Art Rediscovered, Tōkyō, Kodansha International, $176 \mathrm{p}$.

CHONG Pyŏng-mo 鄭炳模, 2005 《Chōsen minga-ron》 朝鮮民画論 (Les discours sur la peinture populaire coréenne) in Pan'gapta! uri minhwa 반갑다! 우리민화 / Ureshii! Chōsen minga うれしい! 朝鮮民画 / Happy! Joseon Folk Painting, Séoul, Sŏul yŏksa pangmulgwan 서울역사박물관, p. 262-271.

6 CHRISTOPHE Jacqueline, BOEll Denis-Michel, meYran Régis, 2009 Du folklore à l'ethnologie, Paris, Éditions de la Maison des sciences de l'homme.

COOLIDGE RouSMANIERE Nicole, 1995 "Yanagi's America: Soetsu Yanagi's Two Extended Stays in the United States and Their Impact on America ", in Mingei. Two Centuries of Japanese Folk Art (Peabody Essex Museum, Joslyn Art Museum, etc.), The Japan Folk Crafts Museum, p. 48-55.

CUISENIER Jean, 1975 L'art populaire en France. Rayonnement, modèles et sources, Fribourg, Office du Livre.

FIGAL Gerald, 1999 Civilization and Monsters: Spirits of Modernity in Meiji Japan, Durham / London, Duke University Press, 304 p.

FLAMMARION Camille, 1907 Les Forces naturelles inconnues, Paris, Flammarion, $604 \mathrm{p}$.

FOISSY-AUFÈRE Marie-Pierre et al., 2000 Mingei de la collection Montgomery. Beauté du quotidien au Japon, Nice, Musée des arts asiatiques.

FROLET Élisabeth, 1986 Yanagi Sōetsu ou les éléments d'une renaissance artistique japonaise, Paris, Publications de la Sorbonne, $214 \mathrm{p}$.

FROLET Élisabeth, 1987 « Yanagi Sōetsu et son mouvement d'art populaire, le mingei undō. Le rôle ambigu de l'Europe et de sa modernité dans la formation d'un mouvement artistique japonais dans les années 30 ", Gazette des Beaux-Arts, t. CIX, 1417 livraison, février 1987, p. 87-96.

24 FURUTANI Yoshiyuki 古谷可由 et al., 2009 Shirakaba-ha no ai shita bijutsu 白樺派の愛した 美術 - 『白樺』誕生100年 (Les œuvres d'art aimées par le groupe Shirakaba. Centenaire de la création de la revue Shirakaba), Ōsaka, Yomiuri shinbun-sha 読売新聞 社.

GORAI Shigeru 五来重, (1966) 1972 Bishōbutsu. Mokujiki no kyōgai 微笑佛一木喰の境涯 (Les bouddhas souriants. La vie de Mokujiki), Kyōto, Tankōsha 淡交社, 243 p.

GORAI Shigeru 五来重, (1968) 1977 Enkū butsu. Kyōgai to sakuhin 円空佛一境涯と作品 (Les bouddhas d'Enkū ». Sa vie et son œuvre), Kyōto, Tankōsha 淡交社, 270 p.

GORAI Shigeru 五来重, 1997 Enkū to Mokujiki 円空と木喰 (Enkū et Mokujiki), Kyōto, Tankōsha 淡交社, $293 \mathrm{p}$. 
GORGUS Nina, 2003 Le magicien des vitrines. Le muséologue Georges Henri Rivière, Paris, Éditions de la Maison des sciences de l'homme.

Heinich Nathalie, 1993 Du peintre à l'artiste. Artisans et académiciens à l'âge classique, Paris, Les éditions de Minuit, $302 \mathrm{p}$.

HeINICH Nathalie, 2005 L'élite artiste. Excellence et singularité en régime démocratique, Paris, Gallimard, 370 p.

HONG Sŏn-pyo 洪善构, 2008 《Chōsen minga no atarashii rikai » 朝鮮民画の新しい理解 (Vers une nouvelle compréhension de la peinture populaire coréenne), Bessatsu Taiyō 別冊太陽 : Kankoku, Chōsen no kaiga 韓国・朝鮮の絵画 (La peinture coréenne), Tōkyō, Heibonsha 平凡社, p. 108-110.

HONMA Masayoshi 本間正義 (dir.), 1973 Enkū to Hashimoto Heihachi 円空と橋本平八 (Enkū et Hashimoto Heihachi), Kindai no bijutsu 近代の美術, vol. 16, Shibundō 至文堂, $106 \mathrm{p}$.

INAGA Shigemi, 1999 "Reconsidering the Mingei Undō as a Colonial Discourse: The Politics of Visualizing Asian "Folk Craft" ", Asiatische Studien, vol. 53, n 2, p. 219-230.

INOUE Enryō 井上円了，1987-2004 Inoue Enryō senshū 井上円了選集 (Euvres choisies d'Inoue Enryō), Tōkyō, Tōyō daigaku 東洋大学, 25 vol.

International Arts \& Crafts, Londres, Victoria \& Albert Museum, 2005.

«Japanese Peasant Painting », Fogg Art Museum. Harvard University. Notes, vol. II, $\mathrm{n}^{\circ} 5$, juin 1930.

KATAGiRI Shūzō 片桐修三 (dir.), 1971 Genshoku Ōtsu-e zufu 原色大津絵図譜 (Album de peintures d'Ōtsu reproduites en couleurs), ōtsu, Ōmi kyōgei bijutsukan Dai-honzan Enman-in monzeki 近江郷芸美術館・大本山円満院門跡, $172+22$ p.

KATAGIRI Shūzō 片桐修三, 1984 Ōtsu-e kōwa 大津絵こう話, Hikone, ōtsu-e bunka kyōkai 大津絵文化協会, $244+53$ p.

39 KANETANI Miwa 金谷美和, 1996 «Bunka no shōhi. Nihon mingei undō no tenji o megutte » 文化の消費 一 日本民芸運動の展示をめぐって (Consommer la culture. Au sujet des expositions du mouvement des Arts populaires japonais), Jinbun gakuhō (Bulletin de la faculté des sciences humaines), $\mathrm{n}^{\circ} 77$, Kyōto, Kyōto daigaku jinbunkagaku kenkyūjo 京都大学人文科学研究所, p. 63-97.

40 KASHIWAGI Hiroshi et al., 2008 Wa: l'harmonie au quotidien. Design japonais d'aujourd'hui, Paris, Maison de la culture du Japon.

KIDA Takuya 木田拓也, 2007 《Yanagi Sōri no dezain to mingei » 柳宗理のデザインと民 藝 (Le design de Yanagi Sōri et le mingei) in Yanagi Sōri. Seikatsu no naka no dezain 柳宗 理－生活のなかのデザイン (Yanagi Sōri. Le design dans la vie quotidienne), Tōkyō kokuritsu kindai bijutsukan, p. 12-14.

42 KIKUCHI Yuko, 1994 « The Myth of Yanagi's Originality: The Formation of Mingei Theory and its Social and Historical Context ", Journal of Design History, $\mathrm{n}^{\circ}$ 7, Oxford University Press, p. 247-266.

3 КІкUСні Yuko, 1997 "A Japanese William Morris: Yanagi Sōetsu and Mingei Theory ", Journal of William Morris Studies, $n^{\circ}$ 12-2, London, William Morris Society, p. 39-45.

4 KІкUСнг Yuko, 2004 Japanese Modernisation and Mingei Theory: Cultural Nationalism and Oriental Orientalism, New York, Routledge Curzon, 309 p. 
KIMURA Shōhachi 木村荘八, 2003 Kimura Shōhachi nikki 木村荘八日記 (Journal de Kimura Shōhachi), Nikkō, Kosugi Hōan kinen Nikkō bijutsukan 小杉放芲記念日光美術 館, 472 p.

KINOSHITA Nagahiro 木下長宏, 1992 Shisō-shi toshite no Gohho. Fukusei juyō to sōzōryoku 思 想史としてのゴッホー複製受容と想像力 (Van Gogh comme élément d'histoire de la pensée. Réception des reproductions et imagination), Tōkyō, Gakugei shorin 学芸書林, $429 \mathrm{p}$.

7 KISHI Fumikazu 岸文和, 2008 Kaiga kōi-ron. Ukiyo-e no puragumatikusu 絵画行為論 — 浮 世絵のプラグマティクス (Théorie de l'acte pictural. Pragmatique de l'ukiyo-e), Kyōto, Daigo shobō 醍酤書房, 2008, 442 p.

KISHIDA Ryūsei 岸田劉生, 1912a 《Jiko no geijutsu»自己の芸術 (Les Arts du soi), Yomiuri shinbun 読売新聞, 17 octobre 1912, p. 5 ; repris dans Kishida Ryūsei zenshū 岸田 劉生全集 (KRZ, CEuvres complètes de Kishida Ryūsei), vol. 1, Tōkyō, Iwanami shoten 岩 波書店, 1979, p. 39-43.

KISHIDA Ryūsei 岸田劉生, 1912b « Omoide oyobi kondo no tenrankai ni sai shite »思い 出及び今度の展覧會に際して (Souvenirs. Autour de cette présente exposition), KRZ 2, Tōkyō, Iwanami shoten, 1979, p. 234-238.

KISHIDA Ryūsei 岸田劉生, 1926 Shoki nikuhitsu ukiyo-e 初期肉筆浮世繪 (Les débuts de la peinture ukiyo-e), Tōkyō, Iwanami Shoten, $150 \mathrm{p}$.

KURATA Hyakuzō 倉田百三, 1917 Shukke to sono deshi 出家とその弟子, Tōkyō, Iwanami shoten, 293 p. Traduction française : Le prêtre et ses disciples, traduit par Kuni Matsuo et Émile Steinilber-Oberlin, Paris, Rieder, 1932, 290 p.

LACHAUD François, 2010 Le vieil homme qui vendait du thé. Excentricité et retrait du monde dans le Japon du XVIII siècle, Paris, Éditions du Cerf, 152 p.

LEACH Bernard, 1960 A Potter in Japan 1952-1954, London, Faber \& Faber, 246 p.

LEACH Bernard, (1978) 1985 Beyond East and West: Memoirs, Portraits and Essays, London, Faber \& Faber, 320 p.

LEROI-GOURHAN André, 1943 Documents pour l'art comparé de l'Eurasie septentrionale, Paris, Les éditions d'art et d'histoire, $97 \mathrm{p}$.

6 LEROI-GOURHAN André, 2004 Pages oubliées sur le Japon, recueil posthume établi par JeanFrançois Lesbre, Grenoble, Éditions Jérôme Million, 500 p.

5 LODGE Oliver, 1909 The Survival of Man: A Study in Unrecognised Human Faculty, London, Methuen, $406 \mathrm{p}$.

Lombroso Cesare, 1909 After Death - What?, Boston, Small Maynard \& Co, 440 p.

LONGENECKER Martha, 2006 Mingei of Japan. The Legacy of the Founders. Sōetsu Yanagi, Shōji Yamada, Kanjirō Kawai, San Diego, Mingei International Museum.

LUCKEN Michael, 2001 L'Art du Japon au vingtième siècle, Paris, Hermann, 350 p.

LUCKEN Michael, 2009 « Une esthétique de la réplication ou comment les fantômes sont à l'œuvre. La peinture de Kishida Ryūsei », Arts asiatiques, tome 64, Musée Guimet / EFEO, p. 79-94.

2 MARQueT Christophe, 2005 «Le regard de Nagai Kafū : une relecture des arts d'Edo au début du Xx $x^{\mathrm{e}}$ siècle », Cipango. Cahiers d'études japonaises, INALCO, nº 12, p. 308-329. 

絵と泥絵一幕末 ・明治庶民画考 (Peinture sur verre et peinture à la gouache. Réflexion sur la peinture populaire à la fin d'Edo et à l'époque de Meiji), Tōkyō, Kawade shobō shinsha 河出書房新社, $188 \mathrm{p}$. « Japon », p. 325-333. 
SATŌ Morihiro, 1996 Edo doro-e: Gaze on Urban Space in Early Modern Japan, Master's Essay, Columbia University, Master of Arts in Liberal Studies, East Asian Studies.

SATō Morihiro 佐藤守弘, 1999 《Topogurafia to shite no meisho-e. Edo doro-e to toshi no shikaku bunka» トポグラフィアとしての名所絵—江戸泥絵と都市の視覚文 化 (Les peintures de lieux célèbres comme topographie. Peintures à la gouache d'Edo et culture visuelle de la ville), Bigaku geijutsugaku 美学芸術学, $n^{\circ} 14$.

SATō Morihiro 佐藤守弘, 2000 « Toshi to sono hyōshō. Shikaku bunka to shite no Edo doro-e » 都市とその表象一視覚文化としての江戸泥絵 (La ville et sa représentation. Peintures à la gouache d'Edo et culture visuelle), Bigaku 美学, $\mathrm{n}^{\circ} 202$.

SHIBUSAWA Keizō 渋沢敬三, 1933《Achikku no seichō» アチックの成長 （Le développement de l'Attic Museum), in Мгунмото Tsuneichi 宮本常一, 1978.

TAKAMURA Kōtarō 高村光太郎, (1950) 1990 Takamura Kōtarō shishū 高村光太郎詩集 (Anthologie des poèmes de Takamura Kōtarō), Tōkyō, Shinchōsha, $241 \mathrm{p}$.

TAKI Seiichi 瀧精一, 1943 Taki Setsuan bijutsu ronshū. Nihon-hen 瀧拙庵美術論集 一 日本 篇 (Recueil d'essais de de Taki Setsuan sur les beaux-arts. Le Japon), Tōkyō, Zayūhō kankōkai 座右宝刊行会, $444 \mathrm{p}$.

TANAHASHI Kazuaki 棚橋一晃 (dir.), 1973 Mokujiki butsu 木喰佛 (Les bouddhas de Mokujiki), Kajima kenkyūjo shuppankai 鹿島研究所出版会, 213 p.

TANAHASHI Kazuaki 棚橋一晃, 1979 Enkū no geijutsu 円空の芸術 (Enkū et son art), Tōkai daigaku shuppankai 東海大学出版会, 202 p.

TANSMAN Alan, 2009 «A Vision of Beautiful Things: Yanagi Sōetsu », in Alan Tansman, The Aesthetics of Japanese Fascism, Berkeley, University of California Press, p. 107-118.

TSCHUDIN Jean-Jacques, 1979 Les Semeurs - Tanemaku hito : la première revue de littérature prolétarienne japonaise, Paris, L'Asiathèque, $170 \mathrm{p}$.

TSUCHIDA Maki 土田眞紀, 2005 "Yanagi Sōetsu to "kindai bijutsushi": “miru" to iu jissen» 柳宗悦と〈近代美術史〉一〈見る〉という実践 (Yanagi Sōetsu et l'« Histoire des arts modernes ». L'art de « voir »), in Taishō-ki bijutsu tenrankai no kenkyū 大正期美術展覧会の研究 (Études sur les expositions d'art de la période Taishō), Tōkyō, Tōkyō bunkazai kenkyūjo 東京文化財研究所, p. 555-574.

87 VIATTE Germain (dir.), 2008 L'esprit mingei au Japon, Actes Sud, Musée du quai Branly, $144 \mathrm{p}$.

WARNER Langdon, 1936 The Craft of the Japanese Sculptor, New York, MacFarlane, 412 p.

WARNER Langdon, 1952 The Enduring Art of Japan, Cambridge, Harvard University Press, $113 \mathrm{p}$.

WARNER Lorraine d'ORÉMIEULX, 1930 « Kōrai Celadon in America », Eastern Art, College Art Association, Philadelphia, vol. II.

WINDELBAND Wilhelm, 1894 Geschichte und Naturwissenschaft, Strasbourg, J. H. Heitz.

YANAGI Sōetsu 柳宗悦，1980-1992 Yanagi Sōetsu zenshū 柳宗悦全集 (YSZ， Euvres complètes de Yanagi Sōetsu), Tōkyō, Chikuma shobō 筑摩書房, 22 vol.

YANAGI Sōetsu 柳宗悦, 1910 «Atarashiki kagaku»新らしき科學 (Une nouvelle science), Shirakaba, vol. 1-6, vol. 1-7, Tōkyō, septembre, octobre 1910 ; YSZ 1, p. 7-62.

YANAGI Sōetsu 柳宗悦, 1911 «Runoā to sono ippa »ルノアーと其の一派 (Renoir et son école), Shirakaba, vol. 2-3, mars 1911 ; YSZ 1, p. 492-500. 
YANAGI Sōetsu 柳宗悦, 1912 《Kakumei no gaka» 革命の畫家 (Les peintres de la révolution), Shirakaba, vol. 3-1, janvier 1912; YSZ 1, p. 543-567.

YANAGI Sōetsu 柳宗悦, 1914 Wiriamu Burēku 立リアムブレーク (William Blake), Tōkyō, Rakuyōdō ; YSZ, 4, p. 9-625

YANAGI Sōetsu 柳宗悦, 1921a “"Chōsen minzoku bijutsukan" no setsuritsu ni tsuite»「朝鮮民族美術館」の設立に就て (À propos de la création d'un Musée des arts populaires coréens), Shirakaba, janvier 1921 (puis publié en anglais dans The Japan Advertiser le 23 janvier); YSZ 6, p. 79-83.

YANAGI Sōetsu 柳宗悦, 1921b «Chōsen minzoku bijutsu tenrankai ni tsuite 》 朝鮮民族美 術展覧會に就て (À propos de l'Exposition des arts populaires coréens), Shirakaba, $\mathrm{n}^{\circ}$ 21, mai 1921, et Yomiuri shinbun, 9-10 mai 1921; YSZ 6, p. 84-88.

YANAGI Sōetsu 柳宗悦, 1922a Chōsen to sono geijutsu 朝鮮とその藝術 (La Corée et ses arts), Tōkyō, Sōbunkaku 叢文閣; YSZ 6, p. 3-167.

YANAGI Sōetsu 柳宗悦, 1922b « Ushinawaren to suru ichi Chōsen kenchiku no tame ni » 失われんとする一朝鮮建築のために (Pour la défense d'une œuvre architecturale coréenne en péril), Kaizō, septembre 1922 ; YSZ 6, p. 145-154.

YANAGI Sōetsu 柳宗悦, 1924-1925 Mokujiki shōnin no kenkyū 木喰上人之研究 (Études sur le vénérable Mokujiki), Josei 女性; Yanagi Sōetsu sensh $\bar{u}$ 柳宗悦選集 (Euvres choisies de Yanagi Sōetsu), Tōkyō.

YANAGI Sōetsu 柳宗悦, 1914 Wiriamu Burēku サリアムブレーク (William Blake), Tōkyō, Rakuyōdō; YSZ, 4, p. 9-625

YANAGI Sōetsu 柳宗悦, 1921a «"Chōsen minzoku bijutsukan" no setsuritsu ni tsuite ”「朝鮮民族美術館」の設立に就て (À propos de la création d'un Musée des arts populaires coréens), Shirakaba, janvier 1921 (puis publié en anglais dans The Japan Advertiser le 23 janvier) ; YSZ 6, p. 79-83.

YANAGI Sōetsu 柳宗悦, 1921b «Chōsen minzoku bijutsu tenrankai ni tsuite 》朝鮮民族美 術展覧會に就て (À propos de l'Exposition des arts populaires coréens), Shirakaba, n ${ }^{\circ}$ 21, mai 1921, et Yomiuri shinbun, 9-10 mai 1921; YSZ 6, p. 84-88.

05 YANAGI Sōetsu 柳宗悦, 1922a Chōsen to sono geijutsu 朝鮮とその藝術 (La Corée et ses arts), Tōkyō, Sōbunkaku 趣文閣; YSZ 6, p. 3-167.

6 YANAGI Sōetsu 柳宗悦, 1922b « Ushinawaren to suru ichi Chōsen kenchiku no tame ni » 失わ扎とする一朝鮮建築のために (Pour la défense d'une œuvre architecturale coréenne en péril), Kaizō, septembre 1922 ; YSZ 6, p. 145-154.

7 YANAGI Sōetsu 柳宗悦, 1924-1925 Mokujiki shōnin no kenkyū 木喰上人之研究 (Études sur le vénérable Mokujiki), Josei 女性; Yanagi Sōetsu senshū 柳宗悦選集 (Euvres choisies de Yanagi Sōetsu), Tōkyō, Shunjūsha 春秋社, vol. 9, 1955, rééd. 1972, 396 p., seize photographies et une carte hors texte ; YSZ 7, p. 5-229.

08 YANAGI Sōetsu 柳宗悦, 1926a 《Nihon mingei bijutsukan setsuritsu shuisho"日本民藝 美術館設立趣意書 (Manifeste pour la création d'un Musée des arts populaires), avril 1926 ; YSZ 16, p. 5-10.

YANAGI Sōetsu 柳宗悦, 1926b « Getemono no bi »下手ものの美 (La beauté des objets du quotidien) ; YSZ 8, p. 3-14.

YANAGI Sōetsu 柳宗悦, 1927 Zakki no bi 䧱器の美 (La beauté des objets usuels), Tōkyō, Kōseikai 工政会, coll. « Mingei sōsho» 民藝叢書. 
111 YANAGI Sōetsu 柳宗悦, 1928 « Kōgei no michi »工藝の道 (La voie de l'artisanat), Tōkyō, Kōdansha, coll. « Kōdansha gakujutsu bunko »講談社学術文庫, 2005. YANAGI Sōetsu 柳宗悦, 1929a «Ōtsu-e no hanashi »大津繪の話 (Propos sur les peintures d'Ōtsu), conférence radiophonique diffusée à Kyōto le 18 janvier 1929; YSZ 13, p. 3-24. YANAGI Sōetsu 柳宗悦, 1929b Shoki Ōtsu-e 初期大津繪 (Les débuts de la peinture d'Ōtsu); YSZ 13, p. 25-175.

114 YANAGI Sōetsu 柳宗悦, 1929c 《Nihon mingei-hin tenrankai mokuroku 》日本民藝品展 覧會目録 (Catalogue de l'exposition d'œuvres d'art populaire japonais), 15-17 mars 1929 ; YSZ 8, p. 357-359.

115 YANAGI Sōetsu 柳宗悦, 1930a "The Peasant Paintings of Ōtsu, Japan », Eastern Art, College Art Association, Philadelphia, vol. II, p. 5-36.

116 YANAGI Sōetsu 柳宗悦, 1930b «A Note on the Pottery Kilns of the Kōrai Dynasty », Eastern Art, College Art Association, Philadelphia, vol. II.

117 YANAGI Sōetsu 柳宗悦, 1931a «Ōtsu-e no waka»大津繪の和歌 (Les poèmes des peintures d'Ōtsu), Kōgei, n² 2, février 1931 ; YSZ 13, p. 177-202.

118 YANAGI Sōetsu 柳宗悦, 1931b «Sashi-e kaisetsu. Minga ni tsuite »挿繪解説一民畫に就 $\tau$ (Commentaire sur les illustrations. À propos de la peinture populaire), Kōgei, $\mathrm{n}^{\circ} 2$; YSZ 13, p. 369-375.

119 YANAGI Sōetsu 柳宗悦, 1931c «Bijutsu to kōgei » 美術と工藝 (Les beaux-arts et les arts industriels), Ōsaka Mainichi shinbun 大阪毎日新聞, 10-12 février 1931 ; YSZ 8, p. 432-436. YANAGI Sōetsu 柳宗悦, 1933a 《 Mingei no shushi »民藝の趣旨 (Le nature du mingei), in Yanagi Sōetsu, Mingei yonjū-nen 民藝四十年 (Quarante années du mouvement des Arts populaires), Tōkyō, 1958, réédité par Iwanami shoten, coll. « Iwanami bunko », 1984, p. 159-173. YANAGI Sōetsu 柳宗悦, 1933b 《Kōgei to bijutsu »工藝と美術 (Les arts industriels et les beaux-arts), Mingei, n² 27, mars 1933 ; YSZ 8, p. 554-570.

122 YANAGI Sōetsu 柳宗悦, 1934a 《Kaiga-ron 》 繪畫論 (Essai sur la peinture), Kōgei, n 37, janvier 1934 ; YSZ 13, p. 382-399, avant-propos p. 769.

123 YANAGI Sōetsu 柳宗悦, 1934b Bi to kōgei 美と工藝 (Le beau et l'artisanat), Tōkyō, Kensetsusha 建設社 ; YSZ 8, p. 283-337. Première publication juin 1928: «Nani o getemono kara manabiuru ka» 何を『下手物』から學び得るか (Que peut-on apprendre des objets du quotidien?), Bungei shunjū 文藝春秋.

124 YANAGI Sōetsu 柳宗悦, 1935 《Mingeikan no seiritsu »民藝館の生立 (La création de la Maison des arts populaires) 1935 ; YSZ 16, p. 52-53. YANAGI Sōetsu 柳宗悦, 1936a Tsukishima monogatari emaki o mite 築嶋繪巻を見て (En observant le rouleau du conte de l'île artificielle), Kōgei, juin 1936 ; YSZ 13, p. 400-405. YANAGI Sōetsu 柳宗悦, 1936b « “Mono” to “koto”»「もの」と「こと」 (Les « choses » et les « faits »), YSZ 9, p. 170-181. YANAGI Sōetsu 柳宗悦, 1936c Folk-Crafts in Japan, translated by Sakabe Shigeyoshi, Tōkyō, Kokusai bunka shinkōkai, 55 p. YANAGI Sōetsu 柳宗悦, 1937a 《Kōgei-teki kaiga »工藝的繪畫 (La peinture artisanale), Kōgei, nº 73, février 1937 ; YSZ 13, p. 423-436. 

272-287. Publishing, $376 \mathrm{p}$.

「ざぜち」のこと (Au sujet des papiers découpés « zazechi »), YSZ 11, p. 424-432.

YANAGI Sōetsu 柳宗悦, 1939 《Nihon mingeikan annai »日本民藝館案内 (Présentation de la Maison des arts populaires), Gekkan Mingei 月刊民藝 (Le Mensuel des Arts populaires), septembre 1939 ; YSZ 16, p. 87-95.

YANAGI Sōetsu 柳宗悦, 1941 《Mingeigaku to minzokugaku » 民藝學と民俗學 (Études sur les arts populaires et études folkloriques), Kōgei 工藝, n 104, juin 1941; YSZ 9, p.

YANAGI Sōetsu 柳宗悦, 1951 《Ōtsu-e gaisetsu »大津繪概説 (Exposé sommaire sur les peintures d'Ōtsu), Kōgei, n 120, janvier 1951, repris dans YSZ, t. 13, 1982, p. 203-221.

YANAGI Sōetsu 柳宗悦, 1954 《Nihon mingeikan»日本民藝館 (La Maison des arts populaires); YSZ 16, p. 171-253.

YANAGI Sōetsu 柳宗悦, 1957a 《Chōsen-ga o nagamete» 朝鮮畫を眺めて (En contemplant une peinture coréenne), Mingei, n 59, novembre 1957 ; YSZ 6, p. 496-499.

YANAGI Sōetsu 柳宗悦, 1957b 《Fushigi-na chōsen minga » 不思議な朝鮮民畫（Les merveilleuses peintures populaires coréennes) ; YSZ 6, p. 500-512.

YANAGI Sōetsu 柳宗悦, 1959 《Chōsen no minga » 朝鮮の民畫 (La peinture populaire coréenne), Mingei, $\mathrm{n}^{\circ}$ 80, août 1959; YSZ 6, p. 513-518.

YANAGI Sōetsu 柳宗悦, 1960a 《Minga ni tsuite » 民畫について (À propos de la peinture populaire), Mingei zukan 民藝龱鑑, vol. 1, Tōkyō, Hōbunkan, $1960 ;$ YSZ 13, p. 502-516.

YANAGI Sōetsu 柳宗悦, 1960b Yanagi Muneyoshi shūkyō senshū 柳宗悦・宗教選集 (Choix d'écrits de Yanagi Muneyoshi sur les religions), Tōkyō, Shunjūsha, 1960-1961.

YANAGI Sōetsu 柳宗悦, 1992 Artisan et inconnu. La beauté dans l'esthétique japonaise, texte de Yanagi Sōetsu adapté par Bernard Leach, traduction française Mathilde Bellaigue, Paris, L'Asiathèque, 165 p.

YANAGITA Kunio 柳田國男, YANAGI Sōetsu 柳宗悦, SHIKIBA Ryūzaburō 式場隆三郎, HigA Shunchō 比嘉春潮, 1940 《Mingei to minzokugaku no mondai »民藝と民俗學の問題 (La question des arts populaires et des études folkloriques), Gekkan Mingei, vol. 2, $\mathrm{n}^{\circ} 4$, avril 1940 ; YSZ 10, p. 735-747.

YeOLSU Yoon, 2003 Folk Painting. Handbook of Korean Art vol. 4, Londres, Laurence King

2 YoshidA Kenji, 2000 "Tōhaku and Minpaku within the History of Modern Japanese Civilization: Museum Collections in Modern Japan ", Senri Ethnological Studies, n 54, Ōsaka, National Museum of Ethnology, p. 77-102.

YosHIDA Shōgorō 吉田小五郎, 1937 《Doro-e no hanashi» 泥繪の話 (Propos sur les peintures à la gouache), Kōgei, n 73, mars 1937. 